\title{
Contribuição dos métodos de imagem na avaliação do envolvimento pulmonar na dermatomiosite ${ }^{(*)}$
}

\section{Contribution of imasing methods in the evaluation of the pulmonary involvement in dermatomyositis}

\author{
Patrícia Noronha Guimarães ${ }^{(1)}$, Fábio Henrique Fernandes Teixeira ${ }^{(2)}$, \\ Ana Paula Monteiro Gomides ${ }^{(1)}$, Marco Aurélio Vignoli ${ }^{(3)}$, Willon Garcia Carvalho ${ }^{(4)}$, \\ Maria Luiza Bernardes Silva ${ }^{(5)}$, Paulo Madureira de Pádua ${ }^{\left({ }^{(6)}\right.}$
}

\section{INTRODUÇÃO}

A dermatomiosite (DM) é uma desordem generalizada caracterizada por miopatia inflamatória e comprometimento cutâneo típico. A miopatia é representada por fraqueza da musculatura proximal das regiões escapular e pélvica, caracteristicamente simétrica, indolor, de início e progressão lentos. No entanto, o grau de inflamação pode variar desde clinicamente inaparente (como na dermatomiosite sem miosite) até casos graves, de início agudo, com acometimento da musculatura intercostal, levando à insuficiência respiratória. O comprometimento cutâneo típico apresenta pápulas de Gottron (patognomônicas), sinal de Gottron, heliotropo, entre outros menos típicos, como eritema linear sobre a superfície extensora das mãos, vasculite periungüeal, hipertrofia das cutículas, sinal do $\mathrm{V}$ ou do xale e as chamadas mãos de mecânicos (associadas à síndrome antisintetase $)^{(1,2)}$.

Outros órgãos internos podem estar envolvidos, dentre eles o pulmão. Há quatro formas de doença pulmonar: alveolite difusa, com tosse e dispnéia rapidamente progressiva, encontrada nos pacientes com síndrome anti-sintetase; uma forma mais lentamente progressiva, em que a miopatia mascara a gravidade do envolvimento pulmonar (a mais freqüente); pneumonia de aspiração e uma forma assintomática na qual há apenas os achados radiográficos $\mathrm{e} / \mathrm{ou}$ funcionais correspondentes à doença intersticial ${ }^{(3)}$. Esta última ocorre em 5\% a 30\% dos pacientes na dependência dos métodos utilizados na investigação ${ }^{(4)}$.

O pneumomediastino é uma complicação rara $^{(5)}$ e está relacionado a pior prognóstico ${ }^{(6)}$. Sua etiopatogênese ainda não foi esclarecida, mas os seguintes mecanismos foram levantados na literatura: vasculopatia pulmonar levando à necrose e ruptura da árvore traqueobrônquica, corticoterapia provocando uma fragilidade tissular, bem como aumentando o risco de infecções e fibrose pulmonar com ruptura de cistos e bolhas subpleurais ${ }^{(7-11)}$.

\section{ASPECTOS RADIOGRÁFICOS}

O estudo das lesões pulmonares é feito com radiografias simples e, principalmente, por meio de tomografia computadorizada (TC) do tórax. O baixo custo da radiografia simples a torna o primeiro exame para a detecção e avaliação inicial das doenças pulmonares intersticiais. Entretanto, em cerca de $15 \%$ dos pacientes com lesão intersticial difusa, a radiografia será normal ${ }^{(12)}$. A TC, por sua vez, apresenta maior sensibilidade e permite o estudo seccional da

\footnotetext{
Santa Casa de Misericórdia de Belo Horizonte.

1. Especializandas do Serviço de Reumatologia da Santa Casa de Misericórdia de Belo Horizonte, MG.

2. Especializando do Serviço de Radiologia da Santa Casa de Misericórdia de Belo Horizonte, MG.

3. Preceptor do Serviço de Reumatologia da Santa Casa de Misericórdia de Belo Horizonte, MG.

4. Preceptor do Serviço de Radiologia e Diagnóstico por Imagem da Santa Casa de Misericórdia de Belo Horizonte, MG.

5. Médica Radiologista da Axial Centro de Imagem, Belo Horizonte, MG.

6. Chefe do Serviço de Reumatologia da Santa Casa de Misericórdia de Belo Horizonte, MG.

Endereço para correspondência: Paulo Madureira de Paula e Patrícia Noronha Guimarães. Santa Casa de Misericórdia de Belo Horizonte, Enfermaria de Reumatologia, 9. ${ }^{\circ}$ andar, ala B, Av. Francisco Sales, 1.111, Funcionários, CEP 30150-221, Belo Horizonte, MG, e-mail: triciapimenta@ig.com.br
} 
anatomia. O uso da técnica de alta resolução na TC (TCAR) proporciona uma melhor avaliação do parênquima pulmonar, tornando possível o diagnóstico de lesões intersticiais em estágio inicial.

Os achados radiográficos da doença intersticial são indistinguíveis dos encontrados na fibrose pulmonar idiopática e em outras doenças do tecido conjuntivo e consistem notadamente em padrão intersticial retículo-nodular simétrico, predominantemente periférico e basal ${ }^{(13)}$.

$\mathrm{Na}$ TCAR evidenciam-se opacidades em vidro fosco, que representam doença pulmonar ativa, e são causadas tanto por lesão intersticial quanto por envolvimento do espaço aéreo. Outros achados de comprometimento intersticial são representados por bandas parenquimatosas, linhas subpleurais e espessamento peribroncovascular e dos septos interlobulares. A fase terminal da doença pulmonar intersticial é caracterizada pelo faveolamento (padrão em "favode-mel"), que pode ser acompanhado de bronquiectasia de tração (Figuras 1a/1b)

Além dos padrões descritos acima, também podem ser observadas áreas de lesão dos espaços aéreos, representadas por consolidação peribroncovascular e subpleural, notadamente em lobos médio e inferiores, associadas a mínimas densidades reticulares. Histologicamente, essas áreas representam bronquiolite obliterante com pneumonia em organização (BOOP) e/ou lesão alveolar difusa (Figura 2).
Mino et al. ${ }^{(4)}$, em um estudo por TCAR de 19 pacientes com comprometimento pulmonar, demonstraram irregularidades pleurais, espessamento septal interlobular, opacidades em vidro fosco e consolidações esparsas em $100 \%$ dos exames, bandas parenquimatosas e espessamento peribroncovascular irregular em $78 \%$ e linhas subpleurais em $36 \%$. Os exames de controle após tratamento comprovaram a reversibilidade dos achados radiológicos, conforme já descrito por Frazer ${ }^{(12)}$ (Figura 3).

O pneumomediastino é uma complicação da doença pulmonar inflamatória ${ }^{(14)}$, e caracteriza-se pela presença de ar nos tecidos mediastinais fora do esôfago, árvore traqueobrônquica ou pericárdio, podendo ser acompanhado por pneumotórax e enfisema subcutâneo. Quando isolado, é usualmente benigno, resolvendo-se normalmente sem a necessidade de procedimentos intervencionistas. Pode ser reconhecido pelo deslocamento lateral da pleura mediastinal por uma coleção aérea que envolve os vasos mediastinais, as vias aéreas principais e o esôfago (Figura 4), diferentemente do pneumopericárdio, em que o ar envolve somente o coração. Outro sinal radiológico importante é o "sinal do diafragma contínuo", no qual é evidenciada a porção central do diafragma (infracardíaco), por causa da interposição de uma coluna aérea entre o coração e o diafragma. Esse é um sinal importante em pacientes acamados (posição supina) $)^{(15)}$.
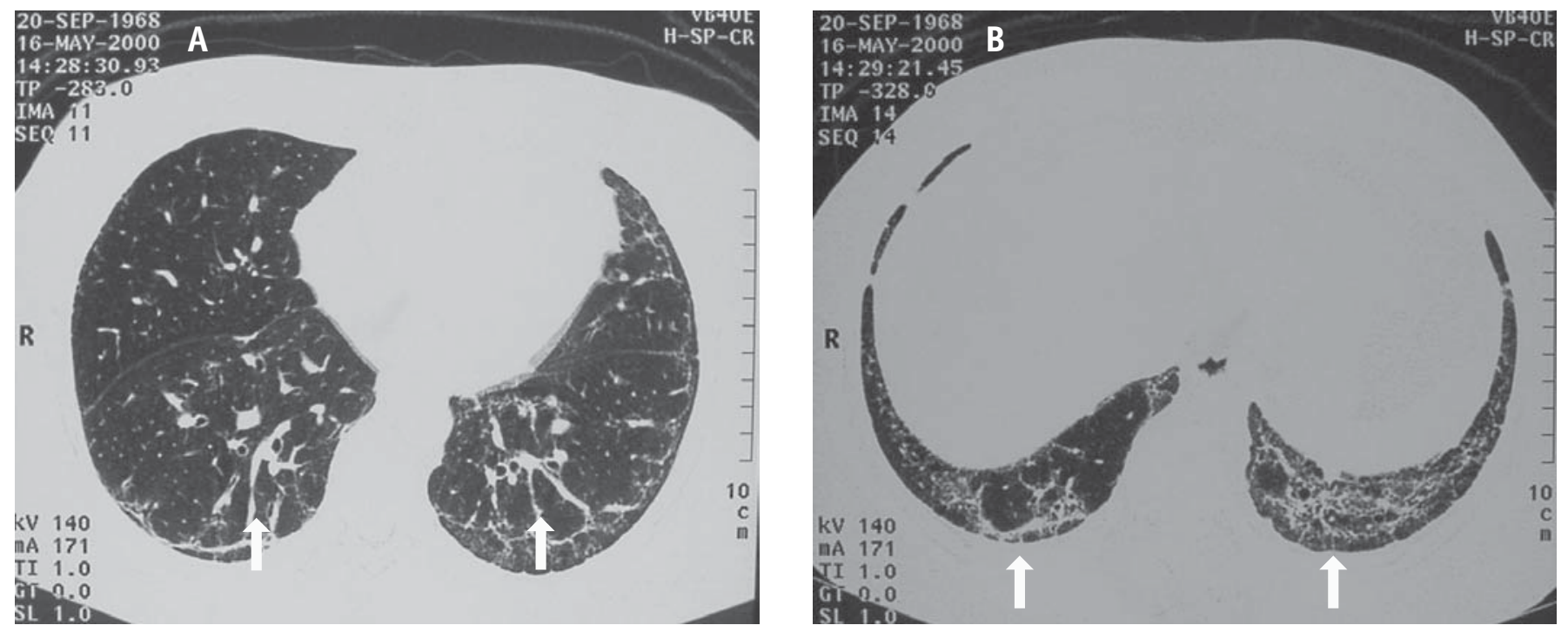

Figuras $1 \mathrm{a} / 1 \mathrm{~b}$ - Tomografia computadorizada de alta resolução de uma paciente com dermatomiosite. Evidenciam-se sinais de fibrose pulmonar intersticial, representados por opacidades reticulares subpleurais (setas), mais pronunciadas em segmentos caudais dos pulmões e por dilatação brônquica de tração no lobo inferior esquerdo. 
O enfisema subcutâneo apresenta-se com freqüência em pacientes com pneumomediastino, sendo evidenciado pela presença de ar em tecidos subcutâneos e pela dissecação das fibras dos músculos peitorais pelo ar (Figura 5).

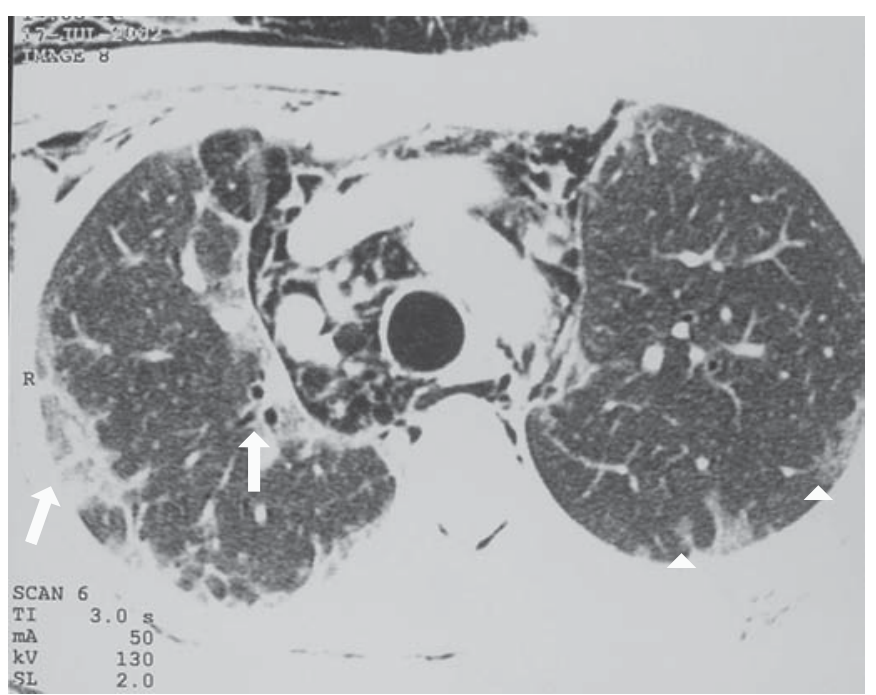

Figura 2 - Tomografia computadorizada de paciente com dermatomiosite. Evidenciam-se consolidações esparsas e periféricas em lobo superior direito (setas). Observam-se também opacidades em vidro fosco à esquerda (cabeças de seta), pneumomediastino e enfisema subcutâneo.

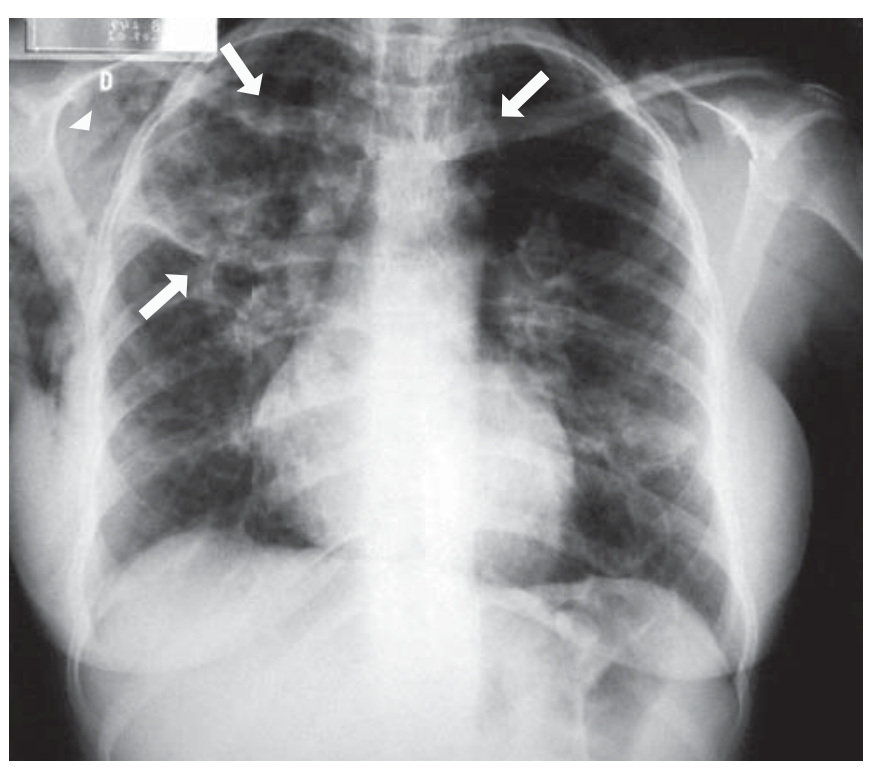

Figura 3 - Radiografia de tórax em PA da mesma paciente da Figura 2. Vêem-se pneumomediastino (setas simples), enfisema subcutâneo (cabeça de seta) e lesões do parênquima pulmonar notadamente em topografia de lobo superior direito.

\section{CONCLUSÃO}

Diante de um paciente com dermatomiosite, queixando-se de dispnéia, é obrigatória uma investigação ativa em busca de uma possível doença pulmonar intersticial, bem como de outras complicações pulmonares menos freqüentes.

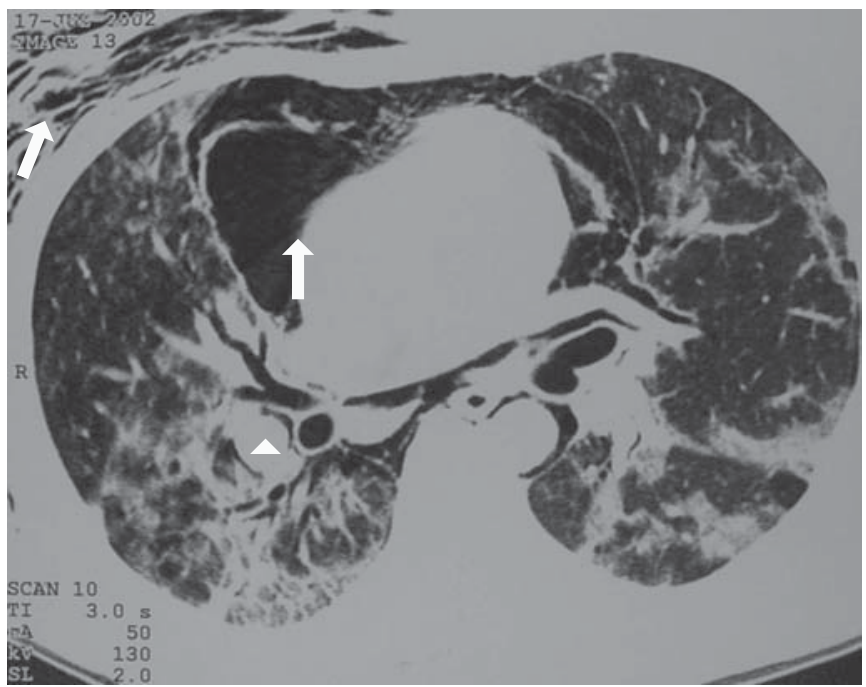

Figura 4 - Tomografia computadorizada da mesma paciente da Figura 2. A paciente apresenta pneumomediastino e enfisema subcutâneo (setas). Observar também presença de menisco aéreo (cabeça de seta) adjacente à artéria interlobar direita, podendo corresponder a enfisema intersticial.

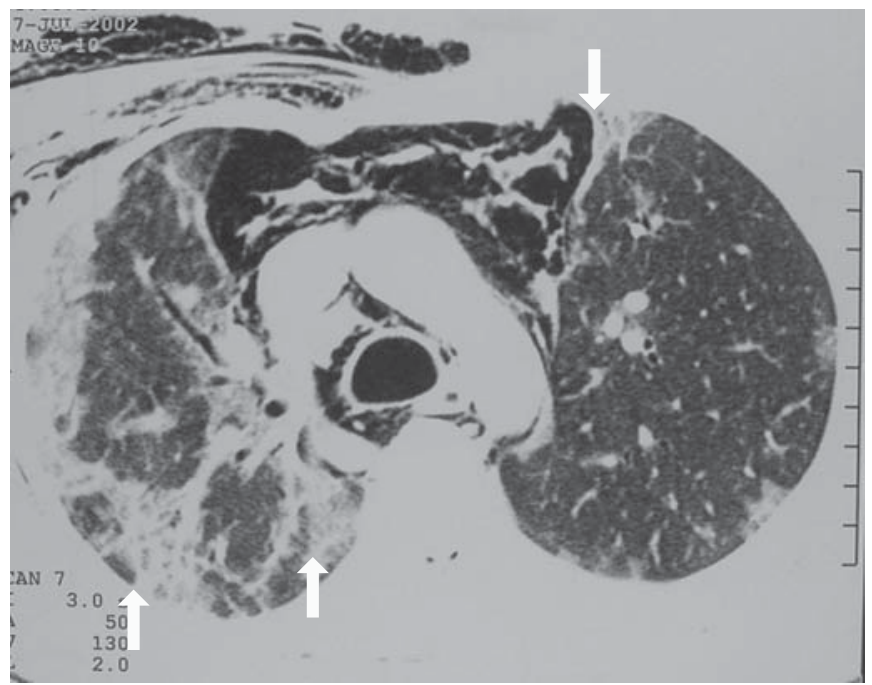

Figura 5 - Tomografia computadorizada. Notar áreas notadamente periféricas com espessamento septal e vidro fosco, representando pavimentação em mosaico (setas), pneumomediastino e enfisema subcutâneo. 


\section{REFERÊNCIAS}

1. Koopman WJ: Arthritis and Allied Conditions. $14^{\mathrm{a}}$ ed. Filadélfia: Lippincott Williams e Wilkins, 2001.

2. Moreira C, Carvalho MAP: Reumatologia - diagnóstico e tratamento. $2^{\mathrm{a}}$ ed. Belo Horizonte: Editora Medsi, 2001.

3. Klippel JH, Dieppe PA: Rheumatology. $2^{a}$ ed. Londres: Editora Mosby, 1998.

4. Mino M, Noma S, Taguchi Y, Tomii K, Kohri Y, Oida K: Pulmonary Involvement in Polymyositis and Dermatomyositis: sequential evaluation with CT. AJR 169:83-7, 1997.

5. Korkmaz C, Ozkan R, Akay M, Hakan T: Pneumomediastinum and subcutaneous emphysema associated with dermatomyositis. Rheumatology (Oxford) 40(4): 476-8, 2001.

6. Hamamoto Y, Takahashi H, Matsunaga T, et al: A case of dermatomyositis associated with asymptomatic pneumomediastinum. Nihon Rinsho Meneki Gakkai Kaishi 21(5):213-9, 1998.

7. Barvaux VA, Van Mullem X, Pieters TH, Houssiau FA: Persistent pneumomediastinum and dermatomyositis: a case report and review of the literature. Clin Rheumatol 20(5):359-61, 2001.
8. Nonomura Y, Koike R, Nishio J, et al: A case of dermatomyositis complicated with pneumomediastinum that was successfully treated with cyclosporine A. Ryumachi 41(3):653-8, 2001.

9. Kono H, Inokuma S, Nakayama H, Suzuki M: Pneumomediastinum in dermatomyositis: association with cutaneous vasculopathy. Ann Rheum Dis 59(5):372-6, 2000.

10. Cicuttini FM, Fraser KJ: Recurrent pneumomediastinum in adult dermatomyositis. J Rheumatol 16(3):384-6, 1989.

11. M'Raihi ML, Dakhlia MS, Chebbi ML, Hamza M, Gharbi B: Pneumomediastinum in deramtopolymyositis. Rev Mal Respir 6(6): 555-7, 1989.

12. Fraser RS, Muller NL, Colman N, Pare PD: Diagnoses of disease of the chest. $4^{a}$ ed. Filadelfia: WB Saunders Company, 1999.

13. Lee JKT, Sagel SS, Stanley RJ, Heiken JP: Tomografia computadorizada do corpo em correlação com ressonância magnética. $3^{\text {a }}$ ed, 2001.

14. Bradley JD: Spontaneous pneumomediastinum in adult dermatomiositis. Ann Rheum Dis 45:780-2, 1986.

15. Meholic A, Ketai L, Lofgren R: Princípios de radiologia torácica, Filadélfia, EUA, Editora WB Saunders Company, 1999. 